\title{
Representaciones sociales sobre la práctica en educación artística en los Profesorados de Artes (música y visuales)
}

Sonia Alzamora
Carina Barroso
Adriana Garbarino
Patricia Cusel
Nidia Doris Forte
Claudia Pechin
Diana E. Santos
(Instituto Superior de Bellas Artes "Municipalidad de Gral. Pico")
(Universidad Nacional de La Pampa. Argentina)

\section{Resumen}

Nos propusimos conocer y vincular las representaciones de docentes y estudiantes sobre las prácticas en educación artística, en aquellos espacios curriculares cuyo formato (1) está orientado a la planificación e implementación de situaciones didácticas de los Profesorados de Artes en Música y Artes Visuales del Instituto Superior de Bella Artes (ISBA).

Como supuesto interviene la consideración que las representaciones de los docentes sobre la educación artística son diferentes según la interpretación del objeto arte y la enseñanza del mismo. Esto se actúa en el aula, con situaciones de desestimación o priorización, tanto en el campo pedagógico como en el artístico.

Es un diseño cualitativo, con estudio de seis casos construidos a partir de la interrelación de los discursos de docentes y estudiantes de cada espacio curricular.

Presentamos el análisis de las representaciones acerca del modelo didáctico desarrollado en cada caso y las valoraciones de los actores así como información acerca de sus condiciones particulares de formación, referencias a la concepción de arte y de educación artística.

El diálogo entre la formación heredada (experiencias previas en educación artística) y aquello por adquirir, permite identificar representaciones que reconstruyen, remodelan y enriquecen cada uno de los abordajes sobre la práctica. Este análisis intenta convertirse en un aporte de las actuales prácticas de formación en el ISBA. 


\section{Palabras clave}

Representación social - Formación docente - Educación artística - Enseñanza y aprendizaje.

\section{Summary}

We aim to know and to link the representations of teachers and students about the practices in arts education curriculum in spaces whose format (1) is aimed at planning and implementing of teaching situations of the Faculty of Arts in Music and Visual Arts Superior Institute of Fine Arts (ISBA, for the initials in spanish).

Involving the consideration that the representations of teachers of art education are different interpretations of the object as art and education of the same. This works in the classroom situations of rejection or prioritization, both in the field of education and the arts.

It is a qualitative design, with study of six cases constructed from the interplay of discourses of teachers and students in each curriculum area.

We present the analysis of representations about the teaching model developed in each case and the valuations of the actors as well as information about their conditions of training, referrals to the conception of art and art education.

The dialogue between the legacy training (previous experience in art education) and what to acquire, to identify representations that rebuild, reshape and enrich each of the approaches on practice. This analysis tries to become a contribution of current training practices in the ISBA.

\section{Key words}

Social representation - Teacher's training - Art education - Teaching and learning.

\section{Introducción}

Los grupos de investigación del ISBA, desde hace cuatro años, se dedican a la indagación de la formación docente que se implementa en la institución. En el proyecto aprobado por el Instituto Nacional de Formación Docente se pretende conocer y vincular las representaciones de docentes y estudiantes sobre las prácticas en Educación Artística, en aquellos espacios curriculares cuyo formato está orientado a la planificación e implementación de situaciones didácticas de los Profesorados de Artes en Música y Artes Visuales del ISBA (Diseño Curricular de ambos Profesorados, 2000, p. 31).

Los objetivos específicos apuntan por una parte a realizar una descripción del/los modelos didácticos desarrollado/s en los espacios curriculares, la identificación de las valoraciones de docentes y estudiantes sobre el/los modelo/s, la formación personal, la vinculación con la educación artística y el objeto arte; y por otra parte, se propone realizar una comparación del modelo 
didáctico implementado con los aprendizajes construidos por los estudiantes sobre la práctica docente.

Como supuesto interviene la consideración que las representaciones de los docentes sobre la educación artística son diferentes según la interpretación del objeto arte y la enseñanza del mismo. Esto se actúa en el aula, con situaciones de desestimación o priorización, tanto en el campo pedagógico como en el artístico.

Los gestos de oficio de los docentes son producto de los esquemas de percepción, de apreciación, de conocimiento (habitus (2)) y esto, en la actuación cotidiana, genera marcas en la formación de los estudiantes, de las cuales aquellos no son conscientes y producen tensiones en el campo de la educación artística. Por su parte, los estudiantes responden a habitus construidos en su historia previa (familiar, escolar y extraescolar) sobre "ser docente de plástica/ música" y sus modos de enseñar y aprender artes. Según sea la visión predominante de estos actores será el encuadre de enseñanza y aprendizaje propuesto y actuado y desde el cual miran a los otros en su evaluación de desempeño; es decir, las representaciones de los docentes formadores sobre la educación artística condicionan el sentido de la práctica del rol profesional que el estudiante realiza durante el Profesorado, a través del/los modelos/s didáctico/s que se implementan. Se produce un entrecruzamiento con las representaciones de los estudiantes, futuros docentes de Artes Visuales y Música, que pueden aceptar u oponer resistencia en distinto grado al proceso de enseñanza y aprendizaje, generando disposiciones diferenciadas ante el modelo didáctico del docente. Según sean las representaciones de los actores sobre la educación artística, se reproducen o transforman el sentido de las prácticas docentes.

Esta investigación se encuadra en una metodología cualitativa. La propuesta consiste en recuperar y armar el entramado de voces de docentes y estudiantes, a través de registros de clases y documentos producidos durante el desarrollo de los cinco espacios curriculares seleccionados. Privilegiamos el estudio de casos por su potencialidad analítica en una escala pequeña dentro de un marco limitado de tiempo y espacio; el producto final es una descripción rica y densa, entendida como completa en los factores considerados en su interacción con la intencionalidad de analizar la situación concreta en estudio (Pérez, 1994).

La propuesta metodológica priorizaba, en el proyecto inicial, la construcción de acuerdos con los docentes y estudiantes de 8 espacios seleccionados (los nombres de los mismos se resguardan por ética). Los mismos se cursan de $2^{\circ}$ a $4^{\circ}$ año de la carrera y en distintos cuatrimestres, por lo cual se propuso trabajar con ellos a medida que se fueran implementando.

Para la construcción de datos, se solicitó a estos actores educativos la realización de registros que reconstruyeran las clases en las cuales participaban en calidad de estudiante o docente. Para completar la información, se 
pretendía analizar las planificaciones didácticas producidas por los estudiantes y las evaluaciones que sobre las mismas realizaran los docentes. Ambas fuentes iban a ser analizadas y posteriormente se realizarían entrevistas con los autores de los mismos para conocer su intencionalidad e interpretaciones de los mismos.

\section{Desarrollo}

Desde los primeros meses del 2008 , los docentes y estudiantes indagados presentaron reticencias ante los requerimientos solicitados (reunión, registros, entrevistas) argumentando problemas de falta de tiempo o entregando informes insuficientes.

En este tiempo, las reuniones de investigación se convirtieron en el lugar de análisis de las situaciones que iban aconteciendo, para tratar de encontrarle un sentido que permitiera reorientar las acciones. Fue así como se plantearon una serie de interrogantes acerca de la resistencia que presentaban los docentes a la propuesta; preguntas sobre los motivos que los llevaban a resistir su colaboración en la investigación.

\section{Primeras interpretaciones}

Desde una mirada que considera que el aspecto institucional se constituye en el interjuego de sus actores a la vez que los constituye a éstos, otorgándoles una determinada identidad y un rol al interior de la misma (Fernández, 2000), se vuelve pertinente el análisis de estas situaciones de resistencia inscribiendo las actitudes de los docentes en el contexto de producción; por esto se construye una interpretación que analiza al sujeto en las relaciones con los otros y con la institución de pertenencia, sin negar los elementos de invención y acción individual en términos de una dialéctica de relación entre lo objetivo y lo subjetivo.

Se centra el análisis en tres perspectivas que son percibidas como intervinientes en este proceso de resistencia: a) la historia institucional del ISBA, b) la relación entre docentes y de éstos con la institución y c) la relación de los docentes con el grupo de investigación (quienes son parte del plantel docente).

A) Son relevantes los cambios originados en las políticas educativas que implicaron la implementación de nuevos diseños curriculares en el 2000 así como la introducción de la investigación como función a desarrollar (campo que carece de tradición en los ISFD). Además, en el Instituto se produjo un cambio de gestión con la consecuente modificación de la política interna y un aumento considerable de docentes del área pedagógica por las características de los nuevos espacios curriculares.

B) La resistencia presentada no está solamente relacionada con el tipo de participación del docente en la construcción del sentido de la práctica en 
educación artística sino con el miedo a ser evaluado y que esta información se devele en el contexto institucional. El modo aceptado por los docentes para involucrarse en esta investigación: una entrevista y no ser observados o registrar sus propias clases, manifiesta un resguardo frente al peligro. En una entrevista, el discurso suele tener una retórica particular de la cual se espera cierto convencimiento, que puede o no tener relación con hechos reales.

C) Los miedos pueden ser provocados por la demostración (por parte del equipo de investigación) de resultados no esperables en la enseñanza y el aprendizaje. Los temores se evidenciarían en la forma de presentación de los registros, cuyo nivel de generalidad y/o fragmentación no favorece el análisis en profundidad de las dimensiones didácticas. Otro dato a tener en cuenta es que el grupo de investigación está conformado por colegas. Este condicionante podría explicar el miedo a ser observado por otro que también participa de la formación. Sin embargo resulta más amenazante que el grupo de investigación sea asociado a una tarea de gestión institucional con funciones evaluativas de su desempeño docente.

\section{Nuevo dispositivo metodológico}

El análisis realizado con auxilio de referentes teóricos tuvo como pretensión la comprensión de la situación existente para re-direccionar la metodología y posibilitar la concreción de la investigación pensada. En consecuencia, el equipo de investigación se enfocó en revisar el sentido de la propuesta presentada, entendiéndolo como "incluirla en una historia, organizarla a través de un significante" (Nicastro, 2006, p. 57). Para ello se definió como primera instancia, proponer a los docentes -al inicio del segundo cuatrimestre de 2008- un trabajo de taller en el cual se expusieran los resultados parciales de la investigación en curso, con la intención de hacer visibles y socializables las problemáticas analizadas en la formación, que fueron inferidas de las entrevistas realizadas a ellos, descubriendo inquietudes comunes y generando un clima de trabajo de mayor confianza y, en consecuencia, más participativo.

El corpus empírico de esta investigación quedó conformado con los datos provenientes de entrevistas a los docentes y estudiantes de 6 espacios curriculares de ambos Profesorados. Se recogen también los datos provenientes del taller realizado con los docentes involucrados.

\section{Presentación de las características de los casos}

Cada caso es construido por quien o quienes ocupa/n el cargo docente del espacio curricular y los estudiantes que acceden a la entrevista. Como nos encontramos con grupos pequeños de estudiantes, se entrevistó a dos por cada espacio a excepción de un caso en que termina una sola persona su 
cursado. La entrevista era voluntaria y se hacía de modo conjunto con ambos integrantes, aclarándoseles que sus docentes conocían acerca de la realización de la misma y del resguardo en el anonimato.

A partir de la interrelación de discursos se construyen los datos de cada caso.

Características de los docentes entrevistados: a) El o la docente (mayormente son mujeres) están a cargo del espacio para una sola carrera, a excepción de un caso que la desarrolla para ambos profesorados; b) Son docentes con diferentes trayectorias formativas en Arte (Música y Artes Visuales) y en Ciencias de la Educación; c) Ingresaron en la institución en distintos momentos (oscilan entre los 2 y 10 años de antigüedad); d) Además de este trabajo, a excepción de un caso, los demás desarrollan su actividad laboral en otros niveles educativos.

Podemos caracterizarlos de la siguiente manera:

Caso 1: Es un espacio de 2do. año del Profesorado de Artes Visuales. La docente proviene de la educación artística, con formación y experiencia en el arte y hace varios años que se desempeña en este cargo. Ella se formó en esta misma institución. Los estudiantes que cursan son 10 aproximadamente y tienen un promedio de edad de 20 años.

Caso 2: Es un espacio de 3er. año del Profesorado de Artes Visuales. La docente tiene formación en Ciencias de la Educación, con una incursión en una academia de dibujo en su infancia. Es docente de este espacio desde hace 3 años y es su única vinculación con la institución. Los estudiantes corresponden a un grupo de 15 aproximadamente y tienen 20-21 años de edad.

Caso 3: Es un espacio compartido por ambos Profesorados, en 3er. año. Son 3 docentes con diferentes perfiles: egresada de Ciencias de la Educación, hace 4 años que trabaja en esta institución donde se desempeña en este espacio curricular y en otro de 2do. año; la otra integrante proviene de la educación artística en Visuales y es el primer año que forma parte de este equipo de trabajo. La tercera integrante es docente de Música, es el segundo año que se desempeña en el equipo y está a cargo de otros espacios del Profesorado de Música. Las estudiantes tienen 20-21 años de edad y forman parte de un grupo reducido de alumnos ya que muchos dejaron de cursar este espacio apenas iniciado el mismo.

Caso 4: Es un espacio de 4to. año del Profesorado de Música. Está a cargo de un docente con formación en educación musical que se desempeña hace varios años en varios espacios curriculares; por otra parte, tiene experiencia como músico. Las estudiantes entrevistadas, de 24 y 25 años de edad, son las únicas cursantes.

Caso 5: Es un espacio de 3er. año del Profesorado de Música cuya orientación es similar al Caso 2 sólo que para esta carrera. La docente es egresada de un Profesorado de Música. Hace 3 años que se desempeña en el Instituto y 
en este espacio es su primer año. Tiene experiencia como artista musical. Los estudiantes entrevistados son los únicos que se encuentran cursando.

Caso 6: Es un espacio de 4to. año del Profesorado de Artes Visuales con orientación similar al del Caso 4. Hay dos docentes a cargo: una de ellas es de Ciencias de la Educación y hace varios años que se desempeña en este espacio que comparte con la otra docente, que es la misma del caso 1. La estudiante entrevistada es la única que termina el cursado.

\section{Encuadre teórico-metodológico}

Nos preocupa la nominación de los conceptos a utilizar para definir las representaciones sobre la actuación docente de los formadores y su interrelación con la recepción de la misma por parte de los estudiantes, ya que el vocabulario utilizado dará cuenta de un encuadre teórico determinado.

Las representaciones sociales configuran una manera de interpretar y de pensar la realidad cotidiana y pertenecen a una actividad mental desplegada por los individuos, constituyendo entonces una relación entre el mundo y el sujeto. Son construcciones realizadas por los sujetos en el marco de un contexto social. La noción de representación social involucra aspectos psicológicos, cognitivos y sociales, puesto que el conocimiento se constituye a partir de las experiencias propias de cada persona, de las informaciones y modelos de pensamiento que recibimos a través de la sociedad e implica asimismo la forma de procesamiento de la información recibida del contexto. Las representaciones así elaboradas, funcionan como un modelo de conducta con el cual el individuo categoriza, jerarquiza y organiza su propio universo y el de las relaciones interpersonales y vínculos grupales (Moscovici, 1986; Jodelet, 1986, 2003).

Las representaciones sociales que sustentan estos discursos son el producto de un proceso de elaboración mental en el que se toma en cuenta la historia de la persona, sus experiencias y construcciones personales (cognitivas y afectivas) y las influencias del contexto socio-histórico-cultural. Cada una de las expresiones discursivas nos habla de esa historia con este objeto que es el arte, la educación artística y la docencia en este campo.

Para referirnos a la relación que se establece entre el docente con los estudiantes, debemos hablar de la enseñanza. Para esto, recurrimos a la elaboración realizada por Fentesmacher $(1989$, p. 5) en este primer momento de análisis exploratorio. Considera que la enseñanza consiste en proporcionarle al estudiante los medios para estructurar sus propias experiencias, "preparar el terreno, o sea, empezar por proporcionar el contenido que el estudiante ha de adquirir. A medida que el alumno empieza a comprender lo que está sucediendo ( $y$ el profesor debe ayudarle a verlo), el alumno se convierte en un estudiante, en una persona capaz de lograr independientemente el acceso al contenido". 
¿Qué forma adopta, a criterio de los actores, esta estructuración de la experiencia pedagógica? ¿Podemos hablar de ésta como de modelos de enseñanza y aprendizaje?

Gimeno Sacristán (1992) entiende al modelo didáctico como un sistema teórico, una estructura cuya totalidad de dimensiones, elementos y relaciones complejas entre los mismos posibilita una mejor comprensión del objeto enseñanza y configura la planificación de alternativas metodológicas. Como modelo esquemático constituye una abstracción teórica y su análisis exhaustivo constituye una explicación de la realidad didáctica.

La utilización del concepto "modelo" puede prestarse a errores de interpretación de nuestra intencionalidad porque el mismo está unido a la idea de norma o patrón que prescribe las prácticas. En este caso, recurrimos a dicha expresión tal como la define Terigi (2008) "para referirnos a una operación intelectual, la modelización, que consiste en una formulación de carácter general sobre la resolución práctica de los aspectos sustantivos de la enseñanza en un determinado contexto didáctico" (3).

Exponemos las representaciones construidas acerca del modelo a partir de la reconstrucción de las prácticas de docentes concretas en interacción con sus estudiantes. Intentamos elaborar consideraciones con cierta especificidad acerca de la enseñanza y el aprendizaje en este particular contexto didáctico, apoyándonos en un análisis del discurso docente y de los estudiantes sobre la enseñanza en los casos analizados y realizando una formulación más general de las problemáticas encontradas.

La atención está colocada en el reconocimiento de los modos de abordaje por parte del docente de los múltiples temas de su campo disciplinario y que se expresa en el tratamiento de los contenidos, su particular recorte, los supuestos sobre el aprendizaje, la utilización de actividades, los vínculos que establece en la clase con las prácticas profesionales involucradas en el campo de la disciplina, el estilo de negociación de significados, las relaciones entre la práctica y la teoría (Litwin, 2008).

Analizamos la percepción sobre los elementos didácticos valorizados por profesores y estudiantes; por ello, se pretende una descripción del modelo a partir de ciertas categorías que aparecen en la actuación en el ámbito de la clase, tal como son interpretadas por los actores. Las mismas son: la concepción sobre el arte en la escuela y el valor del arte en la formación del sujeto; la secuencia de las clases; la intencionalidad explícita sobre el espacio curricular; el desarrollo de las clases en cuanto a objetivos, contenidos, recursos, interacciones con alumnos, actividades, bibliografía, clima.

Caso 1: La docente comenta que las presencias y ausencias de elementos artísticos dentro de la escuela determinan las características estéticas en la misma. Valora que los alumnos conozcan la intencionalidad de las propuestas de enseñanza. Por su parte, los estudiantes rescatan del discurso y actuación de 
esta docente, la necesidad de conocer el sentido de la propuesta implementada en el aula y la apertura de actividades a plantear al sujeto que aprende.

Este sentido asignado por la docente al valor del arte en la escuela, a través de sus apreciaciones, valoraciones y actitud, lo transforma en una experiencia de aula para sus estudiantes con los cuales pretende producir imágenes y que esta construcción requiere de esfuerzo y de varios intentos. Los estudiantes evalúan positivamente la integración de otros lenguajes y otras áreas de conocimiento, para brindar mayores posibilidades de desarrollo.

En el aula se plantean como objetivos lograr sujetos críticos y conscientes de la situación actual, comenzando por el conocimiento de textos y corrientes de pensamiento, haciendo debates sobre la educación artística y propuestas variadas de producciones. La propuesta metodológica consiste en la construcción gradual de un libro que contiene dibujos, grabados y textos que reseñan biográficamente los vínculos de los estudiantes con el área artística en particular en su paso por la enseñanza primaria; reconstruye las vivencias escolares representadas a través de imágenes, los dichos de los autores leídos, la teoría. El trabajo en clase es para el armado del libro así como de lectura y reflexión.

Puede decirse que la concepción teórico-metodológica general sobre la educación artística, se presenta coherente con la implementación de la propia clase, que es observada por los estudiantes como clara y significativa.

Las clases se desarrollan de manera constructiva, el ejercicio del rol docente se ejerce de modo activo con un clima amable y compartido, de respeto de unos hacia otros; donde se integra la propuesta didáctica con un hacer artístico. Esto se presenta con una intencionalidad transformativa de los aprendizajes realizados previamente, son su punto de partida las imágenes incorporadas por los estudiantes y desde allí va armando un nuevo sentido a la enseñanza del arte en la escuela, pensando de modo integrado la educación artística para la formación del rol docente, desde los aportes teóricos que son novedosos para los estudiantes y desde la reflexión constante sobre ellos en sus actuaciones. La docente actúa de acompañante en este proceso para el desarrollo de las posibilidades y obstáculos que van presentando los estudiantes.

Caso 2: En los discursos de la docente y de los estudiantes hay diferencias muy marcadas en lo que respecta a los contenidos abordados: la docente sostiene que trata la didáctica general y específica y los estudiantes perciben como central el aprendizaje de la didáctica general.

La docente menciona contenidos y posturas referidos al arte en la escuela y la enseñanza de ciertos contenidos del lenguaje visual; por su parte los estudiantes, reconocen como único aporte significativo en relación a la didáctica específica el que realizan sus pares en la clase. Pareciese que al no coincidir con los planteos de la docente, los estudiantes la descalifican a la vez que justifican por no ser el arte su campo de formación. 
El tipo de práctica propuesta consiste en planificaciones y simulaciones de clase en tercer ciclo de EGB, lo cual es considerado como la principal actividad del espacio por los estudiantes, que realizan una crítica a dicha metodología, en las cuales sus compañeros teatralizan el rol de alumnos de una escuela y la viven con un fuerte sentimiento de artificialidad. La docente justifica las simulaciones en la escasa confianza de los estudiantes en sus propios aprendizajes previos y el insuficiente tiempo de formación en este espacio que es cuatrimestral.

Ambos actores rescatan la buena relación que se establece en el aula, basada por un lado en la cercanía generacional y por otra en la intencionalidad docente de construir un espacio de reflexión y diálogo. La elección de una bibliografía de corte constructivista por parte de la docente es evaluada por los estudiantes con un sentido de coherencia puesto que rescatan la coincidencia con la actitud pedagógica del debate y el disenso.

Caso 3: En el grupo de docentes se manifiesta una escisión entre el componente pedagógico y el artístico al diferenciarse los campos de conocimiento a enseñar: por un lado lenguaje musical, lenguajes visuales y aspectos de la formación docente. Ellas sostienen desde ese lugar un "acompañamiento" entre los aspectos y consideran positiva esta dinámica. Por su parte los estudiantes perciben escasos "acuerdos académicos" entre los docentes; esto incide en el armado de los distintos momentos de la clase y suele provocarles desorientación en ciertos momentos.

Las intencionalidades de las docentes, tales como la contextualización de las prácticas, desinhibirse frente a un grupo de alumnos, son percibidas y valoradas positivamente por los estudiantes.

Dos cuestiones coincidentes surgen como preocupación en ambos actores: la necesidad de afianzar contenidos disciplinares (de música y visuales) para luego poder enseñarlos en las prácticas docentes y cierto monto de ansiedad generado en los estudiantes por el temor ante las prácticas que realizarán por primera vez en forma individual y con un grupo etáreo cercano al suyo (nivel polimodal).

Como análisis de este caso, puede decirse que hay diferencias al interior del equipo docente en sus apreciaciones sobre el lugar y responsabilidad del arte en la formación del sujeto escolarizado, con el cual deberán relacionarse en las prácticas así como sobre el valor de cada uno de los lenguajes artísticos. Por último, se manifiesta en el discurso docente y de los estudiantes una escisión entre la formación artística y la pedagógica, las cuales no son desvalorizadas una por sobre otra pero se las considera posible de presentarse por separado. Pareciera que se posterga la pretendida integración de campos hasta las instancias de planificación e implementación de las prácticas donde se articularían los conocimientos y se avanzaría en la superación de la tensión teoría-práctica. 
Caso 4: El docente parte del diagnóstico que falta el lugar del arte en la escuela y ante esto propone que se jerarquice como espacio formativo puesto que la música no sólo mejora la calidad de vida sino que se relaciona con las otras asignaturas resultando beneficiosa para el rendimiento escolar de los niños en todas las áreas del conocimiento escolar.

La intencionalidad del espacio está ausente en las voces de las estudiantes pero no desde el profesor que sostiene su pretensión de construir un conocimiento conjuntamente con sus alumnos, desde su rol de guía y de incentivación a la reflexión y la búsqueda continua; además pretende mostrar la relación entre arte y sociedad, por otro lado, y ganar nuevos lugares en la escuela.

El docente hace una propuesta de enseñanza en la cual da por conocidos ciertos contenidos (previos desde el diseño curricular) y él "tira puntas" con consignas muy abiertas, dejando librado a las estudiantes las actividades. Contradictoriamente, al momento de evaluar se presentan tensiones porque el docente pretende un producto que no se logra y no es explicitado según las estudiantes.

Los contenidos suelen ser introducidos a partir de una experiencia perceptiva que busca no condicionar la escucha del estudiante con planteos teóricos previos al encuentro con la experiencia musical, completando la actividad posteriormente con marcos teóricos seleccionados. Las estudiantes reconocen solamente una parte de lo enunciado por el docente; pareciera que de la diversidad de temas y bibliografía presentada, sólo recortan los temas específicos tratados para actividades puntuales como si no reconocieran la intencionalidad ulterior de los mismos.

En esta estrategia se estaría dando el primer paso en la articulación teoría-práctica: las estudiantes adquieren ciertos contenidos a través de la escucha, lecturas, trabajos prácticos, discusión, que se convierten en insumos para fundamentar el proyecto final que llevarán a la práctica. En el proyecto estaría la práctica alimentada desde la teoría que se va estudiando en el cursado; en el contenido del mismo, que es la enseñanza de la música, estaría el componente pedagógico y lo específico del lenguaje. Como contrapartida, las estudiantes relatan las acciones sin poder encontrarles un sentido o criterio de coherencia didáctica.

En este caso puede decirse que aparecen diferencias entre el discurso del docente sobre su propuesta y la interpretación realizada por las estudiantes que perciben una falta de secuencia o de regularidad en las clases que no les permite priorizar los contenidos ni reconocer la intencionalidad de la propuesta o la diversidad de recursos presentados.

Caso 5: La docente critica el lugar relegado de la música en la escuela y la valora positivamente; reconoce una inclinación natural del sujeto en relación a dicha actividad y a la vez señala la contribución en el desarrollo de capacidades cognitivas que sirven para otras asignaturas. Por el modo en el cual describen 
las actividades que desarrollan, podría interpretarse que los estudiantes realizan una valoración positiva de dicha disciplina en el aprendizaje escolar.

Docente y estudiantes coinciden en los contenidos abordados, los estudiantes los resumen como de índole teórica en una primera parte y la segunda, en la que la docente trabaja con actividades que los estudiantes puedan llevar a su práctica posterior.

Las actividades realizadas en el aula brindan elementos para realizar las observaciones, ayudantía y práctica en enseñanza primaria y es en ellas donde los estudiantes subrayan fundamentalmente el aporte de este espacio; las mismas se vuelven significativas para éstos porque se realizan en el marco de la experiencia personal. En este sentido, hay coincidencia en ambos discursos en el planteo de vivenciar aquello que llevarán luego a su hacer como practicantes.

La intencionalidad de la docente es que conozcan las distintas teorías, puedan posicionarse desde la teoría y la práctica y vivencien aquello que van a enseñar, coincide con la apreciación de los estudiantes en cuanto a la coherencia entre qué y cómo se les enseña. Podemos interpretar, entonces, que en este espacio las herramientas didácticas y los contenidos del lenguaje musical son internalizadas por los estudiantes como un todo interrelacionado a partir de una estrategia con un fuerte componente experiencial.

Caso 6: Docentes y estudiante presentan importantes coincidencias en cuanto a la realización de la presentación inicial del espacio, lo cual se efectivizó a través de una propuesta escrita en la cual consta la concepción de enseñanza y de educación artística, los objetivos y una guía detallada y fundamentada de los momentos de trabajo en el año lectivo.

Se presenta como relevante el texto paralelo como medio de vinculación con sus docentes, el proyecto propuesto para el Nivel Inicial y los momentos de armado del mismo, incluso los conflictos y problemas que fue atravesando el grupo inicial, del cual luego queda una sola estudiante.

La bibliografía parte de retomar lo ya estudiado a través de una red conceptual y luego van incorporando nuevas lecturas.

La intencionalidad de las docentes de trabajar el sentido de apertura, de compromiso, despertar la curiosidad y ser crítico se encuentra en la valoración de la estudiante de la propuesta del espacio de escuchar al otro, reflexionar en grupo, analizar los conflictos, proyectar, revisar las propias concepciones y modificarlas, resignificar la mirada sobre los textos ya leídos. Así como en el acompañamiento en la ayudantía y prácticas que a juicio de la estudiante "habla del compromiso del docente".

Con respecto a la conformación del espacio con dos integrantes de distinta formación, la estudiante la califica como "una fusión", donde son importantes ambas personas con sus diferentes formaciones para el trabajo pedagógico que se debe realizar. 
Aquí podría hablarse de un acercamiento entre la propuesta y la implementación del proyecto por parte de las docentes y la comprensión y aprendizaje realizado por parte de la estudiante.

\section{Síntesis de los modelos actuados y vivenciados por los actores}

Del análisis realizado sobre las representaciones acerca de la enseñanza en estos espacios curriculares de los Profesorados del ISBA se identifican dos modelos diferentes a los que se decide nominar "integrado" y "mosaico".

Entendemos como "mosaico" la presencia de un todo armado a partir de planos o partes yuxtapuestas que se presentan separadas por líneas demarcatorias de las fronteras que permiten preservar su autonomía. En este modelo de enseñanza los dos campos disciplinares, el pedagógico y el artístico, serían las partes que se presentan de manera fragmentadas, desde una práctica de convivencia aceptable con el otro campo, interactuando en situaciones puntuales pero resguardando su especificidad. La integración disciplinar y construcción de prácticas en el campo de la educación artística se delega a los estudiantes, futuros docentes de artes.

El otro modelo es de "integración", donde se compone un todo con la unión de las partes. En el proceso de aunar o fusionar las partes, ya no se delimitan como en el modelo anterior sino que, se transforman en un nuevo todo. Este modelo de enseñanza está construido como un espacio superador de las disciplinas originando una nueva estructura de conocimiento. Pareciera ser que no se trata de sumar conocimientos provenientes de distintos campos sino de religar conocimientos organizándolos con sentido integrador; no se trata de desestimar la unidad de cada objeto de estudio sino de ver su múltiples facetas fragilizando los campos disciplinares (Edelstein, 2003).

El Caso 3 sería el mejor ejemplo de mosaico, si bien hay una integración de lenguajes a través de experiencias que le ofrecen a los estudiantes con la intención explícita de actuar como modelos de actividades posibles de ser utilizadas en sus prácticas, en el nivel conceptual las disciplinas se presentan en el mismo tiempo y espacio de manera separada; es decir, guardando en sí mismas su especificidad a través de contenidos, bibliografía y actividades por parte de cada docente integrante del espacio. La pretensión es que los estudiantes construyan una práctica innovadora de educación artística siendo que los modos actuados por los docentes manifiestan una escisión entre lo pedagógico y lo artístico.

El mismo modelo se puede observar en el Caso 2, la docente prioriza contenidos y actividades de formación en didáctica y en la vida escolar. La percepción por parte de los estudiantes de la falta o escasa preparación de la docente para considerar los contenidos del área artística, les hace marcar una clara separación entre ésta y la pedagógica. 
Es diferente el Caso 4. La propuesta de enseñanza, si bien se manifiesta coherente desde el punto de vista del docente en cuanto a la manera de abordar los contenidos y de preparar a sus estudiantes para el ejercicio de la práctica, el criterio de construcción de dicho aprendizaje es percibido por los mismos como desordenado, desestructurado y que no les permite abordarlo con claridad. Aquí se plantean dudas respecto al modelo de enseñanza y aprendizaje porque por una parte tiende a la integración de teoría y práctica, del componente pedagógico y musical en su propuesta didáctica y pedagógica y, por otra parte, se observa un desencuentro con las estudiantes que perciben falta de sentido en las actividades que se proponen en aquélla. Entonces no podemos sostener que responde a un modelo de mosaico así como tampoco el de integración. Es un caso diferente que no podemos conceptualizar. Como modelo de integración se presenta el Caso 1, en el cual la docente resuelve las tensiones de una práctica áulica reuniendo e integrando los elementos de la teoría con un hacer reflexivo, donde el contenido es la educación artística con una metodología del campo artístico. Su propia práctica puede actuar modélicamente aunque no lo manifiesta explícitamente. También entrarían en este modelo el Caso 5 y el 6. En el Caso 6, la participación de las docentes que pertenecen a dos campos disciplinares, se realiza de manera integrada, coordinada, tanto en el abordaje de los contenidos, como de los objetivos, metodología y en la reflexión sobre la práctica. La estudiante percibe que construye su aprendizaje de formación para la práctica, con la propia experiencia formativa del espacio. En el Caso 5, la experiencia áulica propuesta por la docente es vivenciada por los estudiantes como preparatoria y formativa para las futuras prácticas docentes en educación artística; rescatando la coherencia interna de la propuesta hacia ellos y de la docente misma con ellos.

\section{La formación de los docentes y sus propuestas de prácticas para los estudiantes}

A continuación se presentará un análisis de las expectativas, problematizaciones y propuestas de práctica que realizan los docentes. En este apartado, se recurrirá a una diferenciación de los mismos por su pertenencia a campos de procedencia disciplinar: del campo de la educación artística y de la educación, haciendo referencia a los principales perfiles formativos que están presentes en los actores.

Los docentes que tienen una formación básicamente artística, como en los Casos 1, 4 y 5 sostienen apreciaciones sobre los estudiantes similares en cuanto a los requerimientos que ellos hacen y las ausencias que encuentran en estos; sin embargo, esta pertenencia al campo no los lleva a producir las mismas propuestas de enseñanza.

En el Caso 1 se observa una coherencia en su concepción de los estudiantes con la propuesta de enseñanza que elabora para transformar las situaciones 
problemáticas de los estudiantes, las cuales son mencionadas por los demás docentes como dificultad presente en el proceso de enseñanza y aprendizaje. Para este actor, no es un impedimento en el desarrollo de la propuesta sino una condición de partida y una estrategia de desarrollo.

En docentes del campo de la educación (caso 2 y una integrante del caso 3 ), el desencuentro con los estudiantes genera tensiones que traen como consecuencia la distancia con el sentido de la práctica. En estos dos casos, la separación manifiesta entre el campo pedagógico y la formación disciplinar genera una desintegración del conocimiento de la propuesta formativa, ya sea porque el propio docente explicita no tener conocimiento sobre "lo artístico" o porque se trabaja en equipos donde el componente pedagógico se aborda en forma separada de la práctica específica.

Frente a los problemas que persisten en los estudiantes, los docentes recurren a soluciones alternativas que muchas veces tienden a establecer reglas más rígidas; en los Casos 3 y 4 hacen este tipo de propuesta para la instancia de evaluación. Estos docentes solicitan que las condiciones de aprobación estén sujetas a reglas más estrictas a fin de tener respuestas comprometidas y responsables de los alumnos, en el afán de garantizar la lectura bibliográfica, el análisis teórico, la intervención práctica, etc. Estos docentes manifiestan que estas decisiones les asignan una imagen institucional tildando su actuación con valoraciones éticas ("buen docente", "la mala de la película"). De modo diferente, en los Casos 1 y 6 tienden a la transformación de estrategias a fin de revisar conjuntamente con los estudiantes las condiciones de su desarrollo.

Se observa que los docentes realizan diferentes lecturas de sus estudiantes ante las situaciones de prácticas propuestas, enmarcadas en una diversidad de formatos. En los espacios curriculares se realizan propuestas de prácticas cuya pretensión es ser desestructurantes para los estudiantes y pueden desarrollarse como actividades a cumplimentar en el corto plazo durante el cursado del espacio curricular (Caso 2 y 4), o ser de carácter reflexivas en el largo plazo del año del espacio curricular (Caso 1 y 6), intervenciones lúdico-vivenciales para integrar los lenguajes artísticos conjuntamente con análisis teórico y reflexivo sobre la teoría y la práctica (Caso 3), considerar al juego como trabajo artístico vinculado a la práctica (Caso 5).

Las propuestas de acercamiento a la práctica varían desde la ayudantía a un docente de aula (Caso 3 y 5), de simulación como ejercicio ficcional del rol (Caso 2), de observación perceptiva y analítica del campo educativo en el cual realizarán a futuro sus prácticas (Caso 1), al ejercicio mismo del rol docente (Caso 6).

Los docentes formadores llevan adelante distintos modos de acompañamiento y coordinación de la práctica. Desde la presencia del docente en cada momento de práctica (Caso 6) o la ausencia de observación en la mayor parte del proceso para que el estudiante adquiera confianza e independencia (Caso 
3), a la delegación en los estudiantes de la mayor parte de las acciones de desarrollo de esta instancia de formación (Caso 4).

En esta breve exposición tratamos de presentar que hay una diversidad de representaciones en el campo de la educación artística sobre la implementación de las prácticas y sobre el rol de docente formador, aunque debemos aclarar que las mismas están dentro de una gama no muy amplia. Una cuestión que surge como elemento común es el diagnóstico compartido por los docentes sobre los estudiantes que no utilizan los saberes didácticos, psicopedagógicos o psico-sociológicos en sus espacios curriculares y en particular en las instancias de práctica.

La recurrencia de estos discursos analizados y los revisados en la investigación realizada en 2005-06, nos permite hipotetizar que en las representaciones de los docentes en torno a la práctica en educación artística persisten concepciones propias de la hibridación disciplinar, generada no solamente por las características del campo del cual provienen sino también de los habitus de los docentes. En términos de Baillauqués (2005) existen representaciones sobre el oficio y también acerca de su ejercicio por los actores.

Es entre los docentes del campo de la educación que se encuentra con mayor fuerza el discurso que sostiene que al enfrentarse los estudiantes a la práctica, se desvanece la imagen esperanzadora de las virtudes profesionales en el proceso de responsabilidad autónoma; el desconcierto frente a la realidad los hace desacreditar la formación docente y vuelven a modelos tradicionales de enseñanza recuperados de la memoria de la infancia (modelos cercanos a creencias míticas, no de la realidad). La representación del oficio tambalea frente a su "paso al acto".

Esta representación, y a modo de hipótesis lo decimos, suele ir acompañada de un modelo de maestro desesperanzado que el propio docente formador expresa en su hacer profesional (como se mostró al inicio de este ítem en la página anterior) y funciona como referente.

\section{Las valoraciones sobre la formación personal, su vinculación con la educación artística y con el objeto arte}

Las representaciones sociales estudiadas son de dos actores: docentes y alumnos en el espacio del aula y sobre la propuesta de enseñanza y aprendizaje. En primer lugar se expondrá el análisis que da cuenta de las representaciones de docentes y estudiantes sobre la práctica en educación artística y cómo intervienen en ellas la formación personal, la relación con el objeto arte y la valoración de la educación artística.

En esta comparación de contenidos representacionales encontramos separaciones y ligazones, que en términos de Simmel (2001, p. 46, citado en Grimson, Ferraudi Curto y Segura, 2009) "en un sentido tanto inmediato como simbólico, tanto corporal como espiritual, somos a cada instante aquellos que 
separan lo ligado o ligan lo separado". Por un lado, existe un conjunto de operaciones de marcación de límites y umbrales que separan y aíslan ámbitos y prácticas y, por otro lado, se van estableciendo puentes y pasajes entre tales ámbitos separados y diferenciados. Así, pensamos que una vía útil para conocer y caracterizar los modos de vivenciar los modelos docentes es analizando las maneras de distinguir y vincular de los actores sociales.

En esta instancia de la investigación, se identificaron estas operaciones como distancias entre docentes y estudiantes sobre la práctica en educación artística. Las preguntas más específicas apuntaron a desentrañar las distancias entre los discursos del docente sobre aquello que enseña y pretende que aprendan los estudiantes sobre la práctica en educación artística y el sentido asignado a dicha enseñanza por parte de los estudiantes. Este diálogo entre la formación heredada (experiencias previas en educación artística) y aquello por adquirir (en el caso de los docentes, la posibilidad de revisar su propia práctica y para los estudiantes, construir nuevos conocimientos), permite identificar representaciones circulantes que reconstruyen, remodelan y enriquecen cada uno de los abordajes sobre la práctica en educación artística.

Es en los Casos 1, 5 y 6 que analizamos la menor distancia entre los contenidos representacionales de docentes y estudiantes.

En el Caso 1 hay un acercamiento entre docente y estudiantes. La propuesta se presenta con una intencionalidad transformativa de los aprendizajes heredados: su punto de partida son las imágenes incorporadas por los estudiantes y desde allí va armando un nuevo sentido a la enseñanza del arte en la escuela, pensando de modo integrado la educación artística para la formación del rol docente, desde los aportes teóricos que son novedosos para los estudiantes y desde la reflexión constante sobre ellos en sus actuaciones. La docente actúa de acompañante en este proceso para el desarrollo de las posibilidades y obstáculos que van presentando los estudiantes.

Las representaciones sobre el arte, lo artístico, ser docente de artes para este caso son cercanas. El juego jugado por la docente en su historia previa (del cual dimos cuenta en trabajos anteriores: Garbarino, Santos, Barroso, $2006,2007)$ con el arte y ser docente, le permite establecer este vínculo de enriquecimiento mutuo que posibilita este hacer en el aula y en la relación con los estudiantes.

En el Caso 5 hay encuentros entre la intención de la docente de lograr un posicionamiento en relación a las características del aprendizaje pensando en "estrategias adecuadas" para cada nivel del sistema educativo y aquello que los alumnos dicen comprender del sentido de las actividades propuestas para tal fin. Se promueve el juego como estrategia didáctica y en plena articulación con el análisis teórico. Existe una articulación entre teoría, análisis reflexivo y práctica, la cual es vivida como coherencia por los estudiantes. 
Ante las necesidades planteadas por los alumnos, la docente organiza estrategias que transforman el desarrollo de la práctica y promueven la sensación de estabilidad y seguridad en los estudiantes.

La historia previa vinculada al campo del arte le permite acercar el hacer docente a la experiencia artística proponiendo como válido su aspecto lúdico como modo de aprendizaje. La docente se involucra en el juego y participa activamente de la producción. Los estudiantes observan la coherencia entre la postura teórica con la propuesta áulica.

En el Caso 6 la intención de las docentes que se confirma en lo dicho por la estudiante, es de logro de coherencia entre lo propuesta verbal durante la cursada del espacio, el material escrito entregada al inicio del cursado del espacio curricular así como la integración entre el componente formativo pedagógico y el artístico.

Frente a las problemáticas surgidas en el año hay coincidencias entre las docentes y estudiante, que fue un proceso de reflexión, un acompañamiento y trabajo comprometido de ambas partes. El sentido de la propuesta es planteada por las docentes como un proceso de transformación donde se parte de las representaciones del estudiante para trabajar sobre los condicionamientos socio-culturales que influyen en la práctica.

La historia jugada de una de las docentes relacionado con el campo del arte es tomado por la otra docente como referente que le ayuda a conocer las vicisitudes de la educación artística y la coloca en una situación de aprendizaje. También la docente de arte dice estar en una instancia de aprendizaje con la docente del campo de la educación. La actitud pedagógica no sólo se manifiesta con los estudiantes sino también entre docentes.

En estos casos, encontramos que a menor distancia entre las representaciones de docentes y estudiantes sobre la práctica en educación artística:

- menor distancia (o coincidencia) entre la intencionalidad de la enseñanza y la disponibilidad para el aprendizaje;

- $\quad$ menor grado de conflictividad entre las representaciones heredadas y por adquirir;

- $\quad$ menor distancia en la identificación de los estudiantes con la propuesta didáctica implementada;

- mayor disponibilidad de los estudiantes a la búsqueda de soluciones transformadoras en la construcción del rol docente.

En los Casos 2, 3 y 4 encontramos mayor distancia o alejamiento en el contenido representacional de un actor y del otro.

En el Caso 2 observamos alejamiento en la concepción teórico-metodológica de la propia docente que se presenta confusa para los estudiantes, quienes no perciben coherencia entre la intencionalidad de la enseñanza y la propia práctica del docente. La propuesta se presenta con intenciones desestructurantes. 
Ante las dificultades que se perciben en el sistema educativo, la docente y los estudiantes no coinciden en el posicionamiento sobre la defensa del lugar del arte en la escuela. Hay también un alejamiento entre la integración que propone el docente del campo pedagógico con el artístico, en relación a su propia práctica (que es desarrollada como independiente del campo artístico) y lo percibido por los estudiantes. La docente actúa como guía aportando distintas herramientas teóricas para la reflexión sobre la práctica.

Para este docente, las representaciones sobre el arte se ven distanciadas de aquéllas referidas al ser docente de artes. Si bien en su historia previa tuvo experiencias artísticas, el conglomerado de imágenes refiere a aquellos imaginarios sociales que en el campo del arte se desarrollaron históricamente (por ejemplo, relacionar la figura del arte con la "persona loca", referencia ésta que constituyó el sentido común al respecto).

En el Caso 3, que es un espacio curricular para los dos Profesorados y conformado por un equipo de docentes, se observa una escisión entre el discurso del lenguaje visual, el musical y el pedagógico al momento de valorizar la educación artística en la escuela. Los estudiantes observan falta de acuerdos en la propuesta del equipo de docentes.

Cuando abordan la problemática de las prácticas, los estudiantes manifiestan dudas, miedos así como dificultades para desarrollar específicamente los contenidos y esto no se corresponde con lo dicho por las docentes acerca del acompañamiento y seguimiento que hacen a sus estudiantes. Los docentes atribuyen ciertos problemas de la enseñanza a la falta de compromiso de los estudiantes y estos plantean la falta de claridad en las propuestas.

La práctica se presenta de manera "modélica" (como acciones a seguir, a ser reflejadas en el hacer docente). Los alumnos dejan translucir que el modelo propuesto no es el que se pone en acto, que hay incoherencia entre la teoría y la actuación del docente.

En este equipo de docentes son diferentes sus vínculos con el arte.

En el Caso 4, el planteo teórico-metodológico del docente es percibido como desorden por parte de los estudiantes. Las tensiones que se generan por ciertos problemas de comunicación entre ambos devienen en dificultades para vincularse. Tanto el docente como los estudiantes manifiestan problemas relacionados con la comprensión del sentido de las actividades, de las consignas, de la articulación con la teoría, de la responsabilidad asignada, del seguimiento de la práctica donde el docente es percibido como ausente.

La propuesta docente pretende un enfoque sobre el hacer docencia ligada a los paradigmas del arte y la función social que estos promueven. Se observa una postura que acentúa las problemáticas sujetas al hacer artístico en función del hacer docencia. El docente, en relación a su historia jugada, se propone como un artista que enseña, que es artista (o especialista en el lenguaje) como condición para ejercer la docencia. 
En estos casos, podemos decir que a mayor distancia entre las representaciones de docentes y estudiantes sobre la práctica en educación artística:

- $\quad$ mayor distancia entre la intencionalidad de la enseñanza y la disponibilidad para el aprendizaje;

- mayor grado de conflictividad entre las representaciones heredadas y/o por adquirir;

- mayor resistencia de los estudiantes a la aceptación de la propuesta didáctica;

- menor disponibilidad de los estudiantes a la búsqueda de soluciones transformadoras en la construcción del rol docente.

\section{Conclusiones}

En el análisis previo dejamos constancia que son diversas las representaciones de los docentes sobre las prácticas en educación artística y que en las mismas se juega con un peso importante la interpretación que se tiene del objeto arte y su vinculación con el componente pedagógico-didáctico en la formación docente inicial, implementándose modelos de enseñanza y aprendizaje en los cuales aquellos están nítidamente separados o bien avanzan en propuestas de integración.

Es así como los estudiantes van realizando recorridos por modelos que les proponen prácticas diferentes durante sus trayectorias formativas en el ISBA. A las propuestas con las cuales se van encontrando y deben actuar en lo cotidiano del aula, los estudiantes les responden desde sus propias historias formativas previas que los llevan a aceptar o resistir algunas de estas formas de actuación, sin tener total conciencia de esa acción aunque sí de las tensiones que se producen en el campo de la educación artística, como el desencuentro o encuentro entre la formación artística y la pedagógica o la vinculación entre teoría y práctica. A partir de estos registros podemos decir que esas trayectorias van dejando marcas desde las cuales podrán leer, apreciar y quizás actuar luego como futuros profesionales.

Al modelo de enseñanza y aprendizaje que llamamos mosaico, por las separaciones que se manifiestan entre los componentes formativos, le corresponde la mayor distancia en el contenido representacional de docentes y estudiantes y al modelo de integración la menor distancia; observamos sólo un caso que se acercaba a la integración desde la propuesta y se correspondía con una máxima distancia en los contenidos representacionales de los actores.

Este hecho complejo requiere que analicemos la vinculación con los campos de formación de los docentes. En la primera situación presentada (modelo mosaico y mayor distancia), hay docentes que provienen de la educación, si bien un caso es un equipo integrado también con especialistas de los distintos lenguajes; se conformó recientemente, con lo cual no tienen una 
historia previa de acuerdos, de co-formaciones. Esto marca una diferencia con la segunda situación (modelo integrado y menor distancia) en el cual hay un equipo integrado por una docente proveniente del campo de la educación pero que trabaja conjuntamente con una colega del área artística desde hace varios años. Esta segunda situación emerge en los casos en los cuales los docentes provienen del arte o, como se dijo recién, trabajan conjuntamente desde hace varios años.

Es por esto que puede concluirse que en los docentes están presentes distintas valoraciones sobre la enseñanza del arte vinculada a la representación que un campo tiene respecto al otro (arte-educación). Por otra parte, los modelos de enseñanza y aprendizaje, mosaico o integración, son un producto de la representación construida sobre la enseñanza y el aprendizaje organizadas curricularmente en un espacio y no necesariamente sobre el "ser docente de arte", ya que aquí se encuentran elementos en los discursos que no confirman estos modelos actuados; por ejemplo: los Casos 2 y 3 apuntan a un docente coherente y transformador en sus prácticas de educación artística.

No solamente el campo de formación del cual provienen los docentes sino sus habitus sobre el hacer docencia tienen una fuerte incidencia en la concepción de la práctica que implementa y que propone a los estudiantes. En este sentido, la imagen del "ser docente" es una construcción más cercana a la representación del maestro ideal, que incorpora representaciones del "ser alumno" en otros tiempos de escolarización, que a la representación del sujeto formador dentro de un campo determinado. En síntesis, los habitus tienen una fuerte incidencia en la concepción que sustenta la práctica. Esto nos permitiría explicar diferencias entre casos analizados; en algunos de los cuales el docente manifiesta gran interés por transformar estas representaciones y actúa conforme a este objetivo. En otros casos, aunque puede identificarse el problema, no se trabaja en torno a ello. También observamos que, en varias situaciones, ambos actores identifican los problemas pero hacen cargo al otro de la distancia generada a partir de las diferentes intenciones.

Por su parte, los estudiantes acuerdan positivamente con las exigencias e intencionalidades coherentes y significativas de sus docentes y cuando se encuentran con discursos que no se condicen con las prácticas actuadas y solicitadas a ellos, señalan la falta de coherencia o bien desestiman esa parte del trabajo docente.

Planteamos como nueva hipótesis que los actores direccionan distintas representaciones sobre la práctica en educación artística; si bien los estudiantes se encuentran con la presión de la evaluación de sus docentes y por esto actúan lo solicitado casi sin crítica visible, no es una reacción pasiva la que realizan cuando no acuerdan si no que se instala el conflicto, la falta de acuerdo subyacente, la escasa disponibilidad frente al aprendizaje exigido. 
Perrenoud (2005) sostiene que los maestros (y agregaríamos aquí también a los estudiantes) están gobernados por una "lógica natural" (sin plena conciencia sobre la actuación) y dependen en gran medida de la parte menos reconocida y explícita de su operatoria. Cuando las representaciones de los actores en torno a la educación artística dialogan transformando o reproduciendo posicionamientos sobre el sentido de las prácticas; cuando escuchamos los dichos del docente sobre aquello que hace para acentuar o cambiar las problemáticas presentes en la formación docente y lo explicitado por el estudiante respecto de éstas para superarlas o perpetuarlas, podemos observar de qué manera los procesos reflexivos les permiten revertir o continuar con una situación compleja que generalmente es expresada en términos de conflicto.

En estos casos analizados, es interesante destacar que la mitad de los docentes que actuaron como referentes de esta investigación, promueven la transformación y llevan adelante la tarea de reflexionar sobre su propia práctica, lo que se advierte en la estrecha distancia entre sus objetivos y lo que dicen lograr los estudiantes.

Quizás nuestro aporte como grupo de investigación dentro de la institución pueda consistir en orientar la pregunta sobre los esquemas que conforman el habitus actuante y la necesidad que éste se convierta en un analizador de la formación, para comprender "qué favorece y qué inhibe su realización, cómo juega la adhesión de los formadores y sus prácticas de formación" (Perrenoud, 1994, p. 10).

\section{Notas Bibliográficas}

(1) El término formato es utilizado en el Diseño Curricular de los Profesorados en Artes del Instituto Superior de Bellas Artes; refiere a la característica que asumirá en cada espacio curricular el trayecto de la praxis y de investigación.

(2) Habitus remite a la categoría desarrollada por Pierre Bourdieu en su obra, de la cual puede mencionarse como fundacional la escrita con J. C. Passeron: La reproducción, en 1971. El habitus es la interiorización de la exterioridad y la exteriorización de la interioridad. Es un principio generador porque es un sistema socialmente disponible de esquemas de pensamiento, de percepción y de apreciación. Esos esquemas son producto y condición de la posición social ocupada por el agente. Mediante la experiencia cotidiana, con base en una determinada posición social, el agente construye determinadas configuraciones mentales que funcionan como principio de evaluación y clasificación de las cosas del mundo. Las prácticas (preferencias, gustos, estilos, lenguajes, vocación, inclinaciones políticas) están en conformidad con esas configuraciones mentales y con las disposiciones corporales a ellas adecuadas.

(3) Versión digital. Sin dato de página. 


\section{Referencias Bibliográficas}

- $\quad$ Bourdieu, P. y Passeron, J.C. (1971) La reproducción. Madrid, Laia.

- Bourdieu, P. (1992) Espacio social y campo de poder. S/d.

- Bourdieu, P. (1995) Las reglas del arte. Barcelona, Anagrama.

- Edelstein, G. (2003) "Prácticas y residencias: memorias, experiencias, horizontes", en Revista Iberoamericana de Educación, 33. [Versión digital].

- Eisner, E. (1998) El ojo ilustrado. Indagación cualitativa y mejora de la práctica educativa. Buenos Aires, Paidós.

- Fenstermacher, G. (1989) "Tres aspectos de la filosofía de la investigación sobre la enseñanza", en Wittrock, M.C. La investigación de la enseñanza. Enfoques, teorías y métodos. Vol. 1. Barcelona, Paidós.

- Fernández, A. (2000) Poner en juego el saber. Buenos Aires, Nueva Visión.

- Garbarino, A., Santos, D., Barroso, C. y Pereira, S. (2006) "Las representaciones sobre las prácticas en Educación Artística desde la perspectiva de los docentes del Profesorado de Artes Visuales", en CD de II Jornadas Nacionales Prácticas y Residencias en la Formación de Docentes. Universidad Nacional de Córdoba.Córdoba, Argentina.

- Garbarino, A., Santos, D., Barroso,C. y Pereira, S. (2007) "El campo de la educación artística: encuentros y desencuentros representacionales", en CD del IV Congreso Nacional y II Internacional de Investigación Educativa. Universidad Nacional del Comahue, Cipolleti, Argentina.

- Gimeno Sacristán, J. y Pérez Gómez, A. (1992) Comprender y transformar la enseñanza. Madrid, Morata.

- Grimson, A.; Ferraudi Curto, C. y Segura, R. (Comp.) (2009) La vida política en barrios populares de Buenos Aires. Buenos Aires, Prometeo.

- Jodelet, D. (2003) "Primeras Jornadas sobre Representaciones Sociales" (Conferencia). Ciclo Básico Común-Universidad Nacional de Buenos Aires, Argentina.

- Litwin, E. (2008) El oficio de enseñar. Condiciones y contextos. Buenos Aires, Paidós.

- Ministerio de Cultura y Educación de la Provincia de La Pampa (2000). Diseños curriculares de los Profesorados de Arte en Artes Visuales y de Música del Instituto Superior de Bellas Artes "Municipalidad de Gral. Pico". General Pico, La Pampa.

- Moscovici, S. (1986) Psicología Social II. Buenos Aires, Paidós.

- Nicastro, S. (2006) Revisitar la mirada sobre la escuela. Exploraciones sobre lo ya sabido. Rosario, Homo Sapiens.

- $\quad$ Paquay, L., Altet, M.; Charlier, E. y Perrenoud, P. (Coords.) (2005) La formación profesional del maestro. Estrategias y competencias. México, Fondo de Cultura Económica.

- Pérez Serrano, G. (1994) Investigación Cualitativa. Retos e Interrogantes, I. Métodos. Madrid, La Muralla. 
- Perrenoud, P. (1994) "Saberes de referencia, saberes prácticos en la formación de los enseñantes: una oposición discutible". Genéve: Faculté de psychologie e de sciences de l'education \& service de la recherche sociologique [Trad. Interna G. Diker].

- Rockwell, E. (Comp.) (1985) Ser maestro, estudios sobre el trabajo docente. México, SEP/El Caballito.

- Terigi, F. (2008) "La enseñanza en las secciones rurales múltiples: problemas identificados y desafíos para la formación", en CD del Congreso Metropolitano de Formación Docente. Facultad de Filosofía y Letras, Universidad Nacional de Buenos Aires. 